\title{
Abstract: Simultaneous Estimation of X-ray Back-scatter and Forward-scatter using Multi-task Learning
}

\author{
Philipp Roser ${ }^{1,4}$, Xia Zhong ${ }^{2}$, Annette Birkhold ${ }^{3}$, Alexander Preuhs ${ }^{1}$, \\ Christopher Syben ${ }^{1}$, Elisabeth Hoppe ${ }^{1}$, Norbert Strobel ${ }^{5}$, \\ Markus Kowarschik ${ }^{3}$, Rebecca Fahrig ${ }^{3}$, Andreas Maier ${ }^{1,4}$ \\ ${ }^{1}$ Pattern Recognition Lab, Friedrich-Alexander-Universität Erlangen-Nürnberg \\ (FAU), Germany \\ ${ }^{2}$ Diagnostic Imaging, Siemens Healthcare GmbH, Erlangen, Germany \\ ${ }^{3}$ Advanced Therapies, Siemens Healthcare GmbH, Forchheim, Germany \\ ${ }^{4}$ Erlangen Graduate School in Advanced Optical Technologies (SAOT), Germany \\ ${ }^{5}$ Institute of Medical Engineering, University of Applied Sciences \\ Würzburg-Schweinfurt, Germany \\ philipp.roser@fau.de
}

Scattered radiation is a major concern that affects X-ray image-guided procedures in two ways. First, in complicated procedures, backscatter significantly contributes to the patient's (skin) dose. Second, forward scatter reduces contrast in projection images and introduces artifacts in 3-D reconstructions. While conventionally used anti-scatter grids improve image quality by blocking X-rays, its attenuation must be compensated by a higher input dose. When quantifying the skin dose, backscatter is usually considered by applying predetermined scalar backscatter factors or linear point spread functions to the patient's skin entrance dose. However, since patients have different shapes, the generalization of conventional methods is limited. Here, we propose a novel approach that combines traditional techniques with multi-task learning to estimate the forward and backscatter simultaneously. In a simulation study including head and thorax data, we jointly estimated forward and backscatter with $94 \%$ accuracy on average and outperformed the associated single-task approaches. In the future, the inclusion of a first-order scatter estimate based on the patient model is a promising approach to increase the overall performance and the physical plausibility [1].

\section{References}

1. Roser P, Zhong X, Birkhold A, et al. Simultaneous estimation of X-ray back-scatter and forward-scatter using multi-task learning. Proc MICCAI. 2020 October; p. 199-208. 